\title{
Elimination of gaseous emissions of the absorption process in formalin production by integrating a vortex-type direct-contact condenser into a traditional process flow scheme
}

\author{
Leonid Moskalev ${ }^{1, *}$, and Sergei Ponikarov ${ }^{1}$ \\ ${ }^{1}$ Kazan National Research Technological University, 420015, 68 Karl Marx Street, Kazan, Russia
}

\begin{abstract}
Currently, special consideration is given to environmental issues. Atmospheric pollution by gaseous emissions from industrial enterprises is caused by a variety of reasons, such as incomplete product yield, peculiarity of reaction behavior, or emissions resulting from the loss of the final product. This article describes gaseous emissions from the operation of the absorption plant for the production of industrial formalin. Various techniques for purifying gaseous emissions are shown. A modernized technological scheme model with an integrated vortex-type direct-contact condenser for absorbing methyl alcohol and formaldehyde vapors from contact gas is developed in the universal simulation package. This model enables the technological modes of industrial formalin production absorption processes to be selected. It has been revealed that the use of a vortex-type direct-contact condenser will minimize atmospheric emissions and increase resource efficiency. The magnitude of the difference between production data and calculated results for the proposed model turned out to be no more than $8 \%$.
\end{abstract}

\begin{abstract}
Almost all chemical and petrochemical enterprises produce gaseous emissions, and pollute the environment. Thus, there is an urgent need to eliminate, neutralize, or dispose the above-mentioned emissions. Nowadays, various methods have been developed for cleaning and gathering gaseous emissions in order to bring the content of toxic impurities to the maximum permissible concentrations [1,2]: dry gas purification (adsorption), catalytic neutralization, afterburning, and wet gas purification (absorption).

This article focuses on the emissions from the operation of the absorption plant for the production of industrial formalin. The amount of absorption gases is $20198 \mathrm{~kg} / \mathrm{h}$, which include toxic substances, such as formaldehyde $(20.2 \mathrm{~kg} / \mathrm{h})$, and methyl alcohol vapors $(20.2 \mathrm{~kg} / \mathrm{h}$ expressed in terms of methyl alcohol). The neutralization of these gases is carried out by flaring. These so-called emissions, in addition to valuable components (methyl alcohol and formaldehyde), contain nitrogen $(18040 \mathrm{~kg} / \mathrm{h})$, and water, which
\end{abstract}

\footnotetext{
${ }^{*}$ Corresponding author: lejnya@yandex.ru
} 
reduce the flame temperature. Therefore, the combustion of absorption gases without special afterburning devices is a difficult process. Thus, formaldehyde and methyl alcohol do not burn completely in the flare stack, and go into the atmosphere in the form of various compounds, for example, methanediol.

To eliminate gaseous emissions, firstly, it is necessary to reduce or eliminate formaldehyde and methyl alcohol vapors before burning in the flare stack. And, to the extent reasonably practical, it is required to return these valuable components to the start of the entire technological chain of industrial formalin production. Such modernization of the process flow scheme of industrial formalin production will lead to both the elimination of these gaseous emissions and the conservation of raw materials. It is possible to reduce the amount of toxic substances in absorption gases by increasing the pressure in the absorber. But the pressure increase will provoke the adverse reactions involving the deposition of formaldehyde resulting in a growing freezing depth. Therefore, it is recommended to use a vortex-type direct-contact condenser [3] for condensation of methyl alcohol vapors and formaldehyde capture. $[4,5]$. The proposed method will allow the main part of unreacted substances to return to the main process through condensation and deentrainment of methyl alcohol and formaldehyde vapors. [5].

However, their widespread use is constrained not only by the need to select a condensing agent, but also by the increased drop entrainment. However, the most significant advantages of mixing condensers over surface condensers are the intensity of heat and mass transfer processes, low metal consumption, simple design, considerable increase of the heat transfer coefficient, and small dimensions. The intensity of the working fluid interaction process is achieved not only by selecting certain physical and chemical characteristics, but also by creating the most favorable hydrodynamic conditions, which can significantly increase the heat removal from the apparatus volume unit. [6, 7]. Among the existing mixing condensers, cyclone and vortex-type condensers have the maximum efficiency and small dimensions in comparison with the parallel-current mixing condensers.

Errors and shortcomings when conducting full-scale experiments at real manufacturing sites come at a cost. Therefore, it is almost impossible to conduct any kind of research there. Accordingly, today more and more attention is paid to computer-based simulation methods that can reduce financial costs, and speed up the design and manufacture process of experimental stands, installations, and equipment. Based on a virtual prototype, it is possible to optimize the design of devices and technological schemes. The ChemCAD universal simulation package can serve as one of such simulation methods. [4].

A vortex-type direct-contact condenser is synthesized in the ChemCAD universal simulation package. [8]. To verify this model, the authors of this article have created an experimental setup that enables physical experiments to be carried out in the process of contact condensation with swirling flows. The calculation results in a vortex-type directcontact condenser synthesized model have been compared with the results of physical experiments. It has been revealed that the experimental error in the calculation is no more than $8 \%$, which is permissible [6].

In addition, a model of the modernized technological scheme of the existing absorption unit for the production of industrial formalin with an integrated vortex-type mixing condenser is built in the ChemCAD universal simulation package. [9]. The synthesized process flow scheme for the production of industrial formalin with a vortex-type directcontact condenser is shown in the Fig.1.

The characteristic of the equipment of the scheme model is similar to the characteristic of the current installation. The model is a set of modules that are interconnected by flows. The design of the model of methyl alcohol and formaldehyde vapor absorption from contact gas with and without the integrated vortex-type direct-contact condenser was carried out using the same mathematical model. However, the vortex-type direct-contact condenser is 
non-standard equipment and, accordingly, there is no module of such condenser in the universal simulation package. Therefore, the user-defined function was written for the universal simulation package. A complete technological scheme design was carried out using mathematical models: NRTL (equilibrium constant global model) and Lee-Kesler (global enthalpy model). The indicated mathematical models take into account heat and mass transfer, and they are available in the database of the universal simulation package; they most closely approximate the calculated data to the production data.

The adequacy of the proposed model of the technological scheme was checked by comparing the following aspects: production and estimated material balances; component composition of production and estimated results regarding methyl alcohol and formaldehyde emissions. Comparison of production and estimated material balances illustrated the difference: absorption gases $-0.046 \%$, industrial formalin $-2.935 \%$. The component-wise comparison demonstrated the difference for absorption gases: methyl alcohol $-1.85 \%$, formaldehyde $-0.23 \%$, other substances - less than $1 \%$; for industrial formalin: methyl alcohol $-4.07 \%$, formaldehyde $-5.92 \%$, water $-10.08 \%$, formic acid $2.04 \%$.

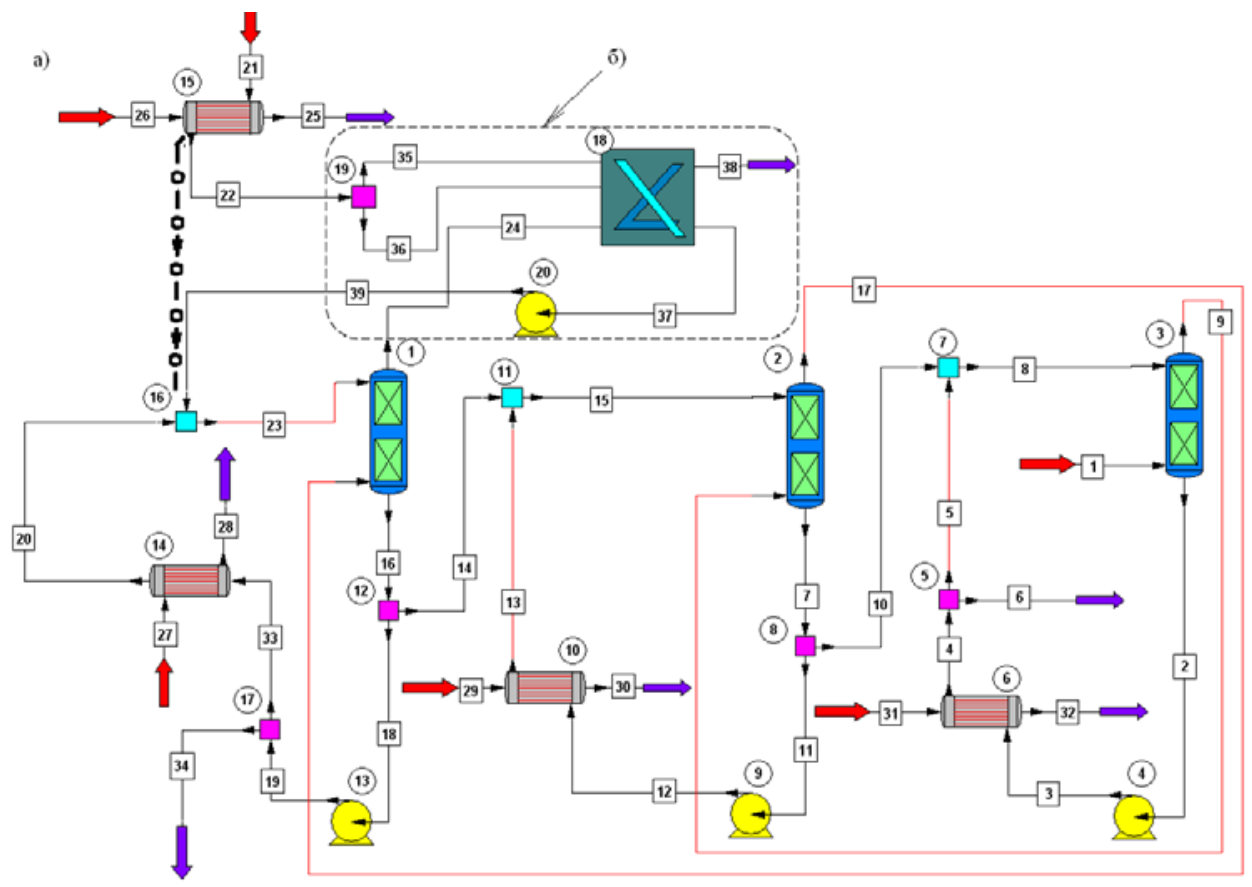

Fig. 1. Process flow sheet synthesized using the universal simulation software package for an existing industrial formalin production plant: (a) conventional process flow sheet $(\rightarrow \mathbf{O} \rightarrow$ is the supply of cooling fluid, flow 22), (b) conventional process flow sheet with a contact vortex condenser (the supply of cooling fluid, flow 22), (1)-(3) absorber (minimum number of theoretical trays), (4), (9), (13), (20) pumps, (5), (8), (12), (17), (19) splitters (for the splitting of flows), (6), (10), (15) coolers, (7), (11), (16) mixers (for the mixing of flows), (14) evaporator, (18) vortex-type direct-contact condenser. Flows: (1) feed of absorber (3) (contact gas), (6) industrial formalin, (21) fresh water flow (additional feeding), (24), (38) absorption gas, (27) liquid ammonia (at a negative temperature), (26), (29), (31) direct water (for cooling), (34) water with a small content of compounds incorporated into a contact gas.

The design of the model of the modernized process flow scheme of the existing absorption unit for the production of industrial formalin with an integrated vortex-type mixing condenser illustrated the following: 
1. The amount of methyl alcohol in absorption gases will decrease by a factor of 3 (Fig.2 dependence of the methyl alcohol content in absorption gases on the temperature of contact gas entering the absorber of the existing installation for the production of industrial formalin without a vortex-type direct-contact condenser (curve 1) and with a vortex-type direct-contact condenser (curve 2);

2. The amount of water for atomization in the absorber will decrease by $30 \%$ of the base amount of water (Fig. 3 - dependence of the methyl alcohol content in absorption gases on the flow rate of the coolant fluid in the absorber of the existing installation for the production of industrial formalin without a vortex-type direct-contact condenser (curve 1) and with a vortex-type direct-contact condenser (curve 2);

3. Using the proposed condenser, formaldehyde will react almost completely (Fig.4 dependence of the formaldehyde content in absorption gases on the temperature of contact gas entering the absorber of the existing installation for the production of industrial formalin without a vortex-type direct-contact condenser (curve 1) and with a vortex-type direct-contact condenser (curve 2) and Fig.5 - dependence of the formaldehyde content in absorption gases on the flow rate of the coolant fluid in the absorber of the existing installation for production of industrial formalin: without a vortex-type direct-contact condenser (curve 1) and with a vortex-type direct-contact condenser (curve 2);

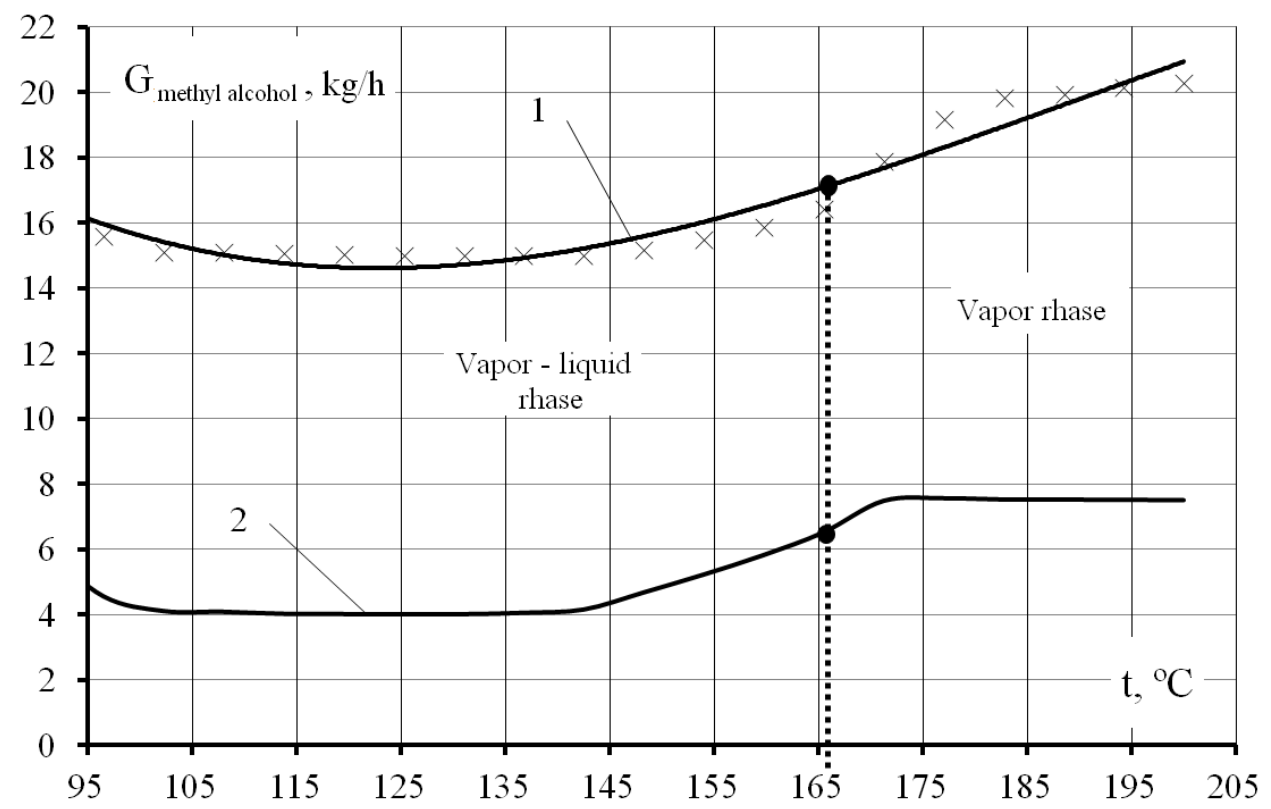

Fig. 2. Dependence of the methyl alcohol content in absorption gases on the temperature of contact gas entering the absorber: $x$ - production data, curve 1 -design and calculation based on the NRTL and Lee-Kesler models (without a vortex-type direct-contact condenser), curve 2 - design and calculation based on the NRTL, Lee-Kesler models, and model for the vortex-type direct-contact condenser. 


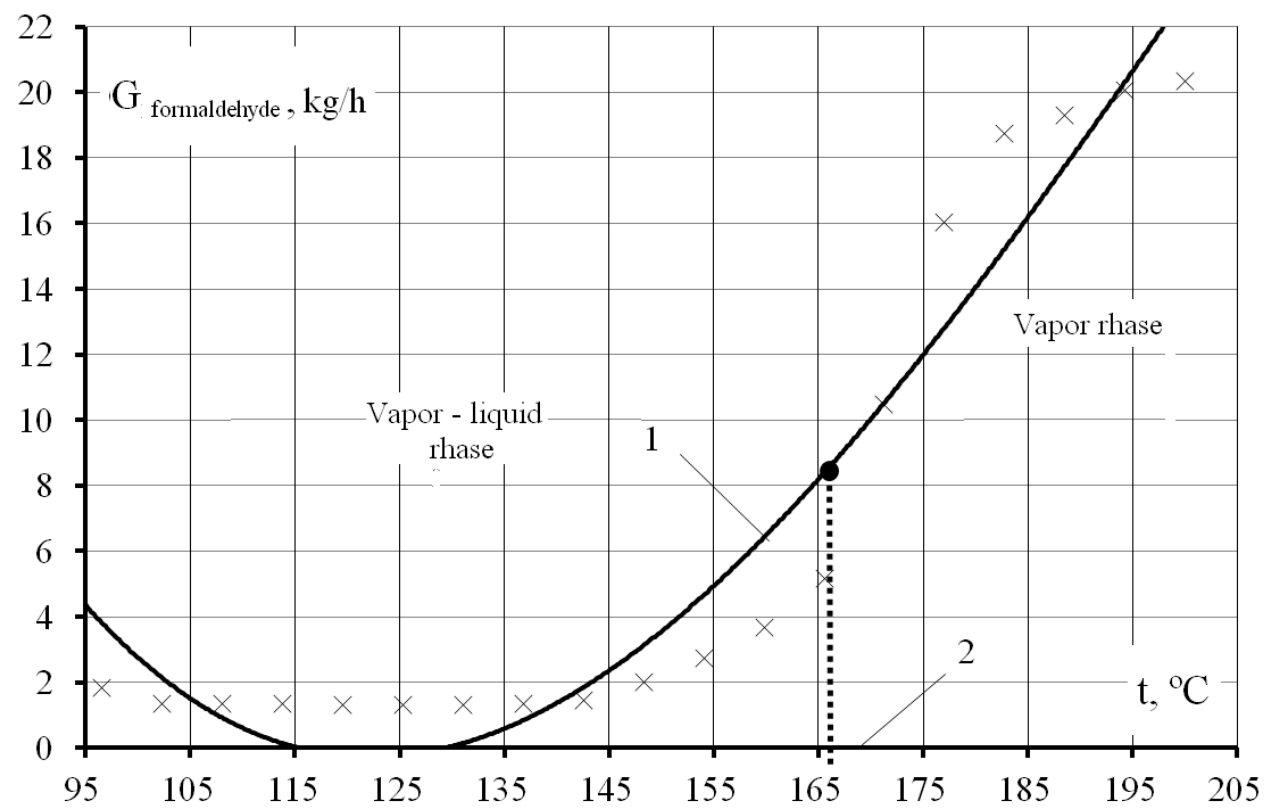

Fig. 3. Dependence of the formaldehyde content in absorption gases on the flow rate of the coolant fluid for atomization in the absorber: $\mathbf{x}$ - production data, curve 1 - design and calculation based on the NRTL and Lee-Kesler models (without a vortex-type direct-contact condenser), curve 2 - design and calculation based on the NRTL, Lee-Kesler models, and model for the vortex-type direct-contact condenser.

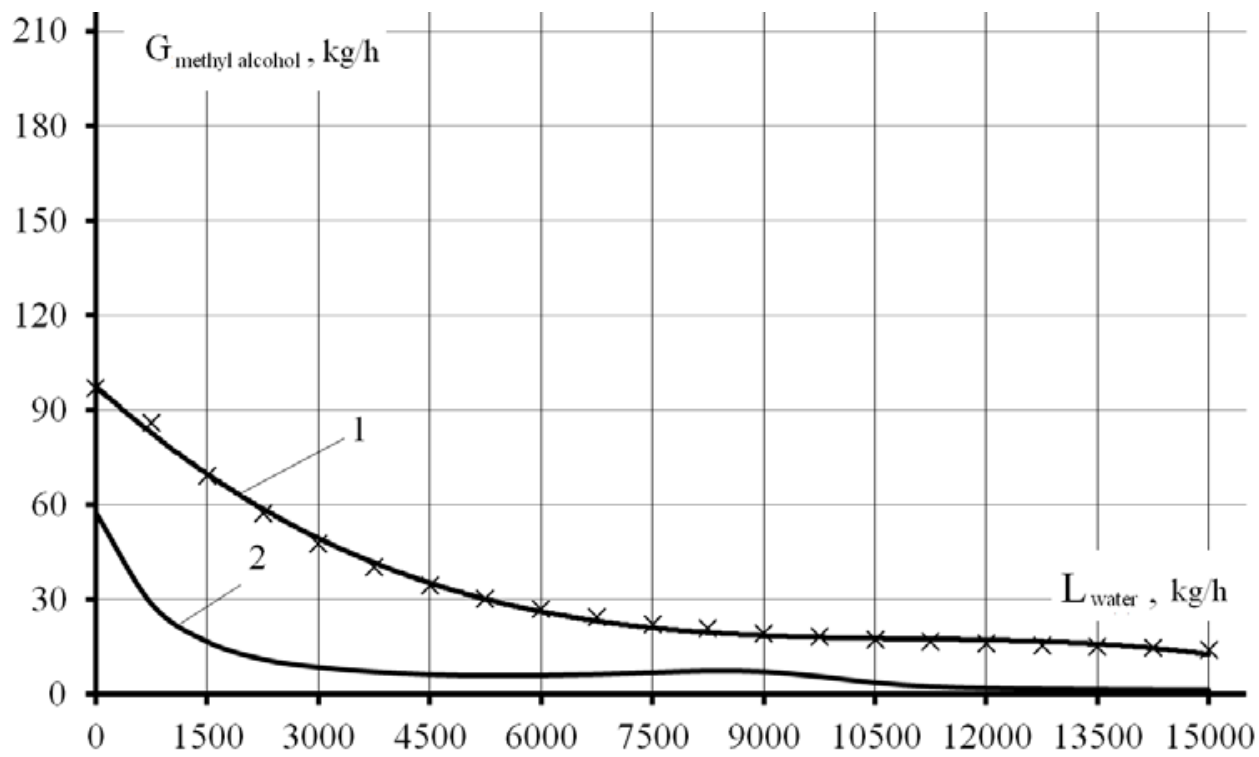

Fig. 4. Dependence of the methyl alcohol content in absorption gases on the temperature of contact gas entering the absorber: $x$-production data, curve $1-$ design and calculation based on the NRTL and Lee-Kesler models (without a vortex-type direct-contact condenser), curve 2 - design and calculation based on the NRTL, Lee-Kesler models, and model for the vortex-type direct-contact condenser. 


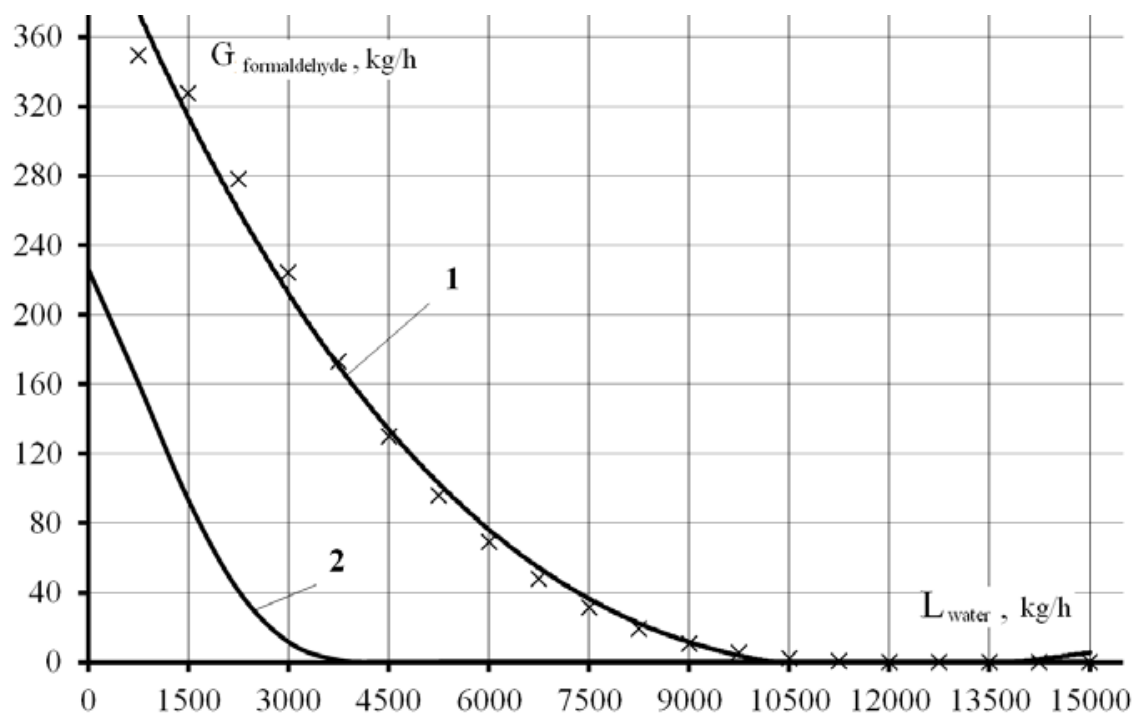

Fig. 5. Dependence of the formaldehyde content in absorption gases on the flow rate of the coolant fluid for atomization in the absorber: $\mathrm{x}$ - production data, curve 1 - design and calculation based on the NRTL and Lee-Kesler models (without a vortex-type direct-contact condenser), curve 2 - design and calculation based on the NRTL, Lee-Kesler models, and model for the vortex-type direct-contact condenser.

It can therefore be concluded that the process flow scheme using a vortex-type mixing condenser is much more efficient. An industrial formalin production plant model developed in the universal simulation package expands opportunities for selecting technological modes and equipment without extra financial expenses.

\section{References}

1. R.H. Muhutdinov, R. Y. Amir, L. E. Almeev, M. Khannanov, The effectiveness of the implementation of vortex systems (for petrochemical production) / Under the General editorship of J. S. Amirov. - Ufa: Publishing House "Reagent", (2001).

2. GN (Hygienic Regulations) 2.2.5.686_98: Threshold Limit Values (TLV) for Hazardous Substances in Work-place Areas), Moscow: Minzdrav Rossii, (1998).

3. I.I. Ponikarov, S.I. Ponikarov, V.V. Alekseev and L.N. Moskalev, RF Patent 2502929, Byull. Izobret., no. 36 (2013,).

4. L.N. Moskalev, , S.I. Ponikarov, Application of a Contact Vortex Condenser To Absorb Methanol from a Contact Gas, Theor. Found. Chem. Eng., v. 50(2), - pp. 194-200 (2016).

5. L.N. Moskalev, S.I. Ponikarov, Use of a Vortex-Type Contact Condenser in Absorption of Methanol and Formaldehyde from a Contact Gas, JEPTER, v. 89(5), - pp. 11791186 (2016)

6. L.N. Moskalev, S.I. Ponikarov, The process of contact condensation in a vortex type mixing apparatus: Monograph, Kazan: KNRTU, p.157 (2018).

7. E.I. Taubman, V.A. Gornev, V.L. Meltzer and other Contact heat exchangers, M .: Chemistry, p. 256 (1987). 
8. L.F. Akhmadeeva, L.N. Moskalev, E.V. Osipov, I.I. Ponikarov,, Description of vortex heat and mass transfer apparatuses using the CHEMCAD 5.2 simulation software, Vestn. Kazan.Tekhnol. Univ., v. 15(11), pp. 158-162 (2012). 